\title{
Perinatal death audits in a peri-urban hospital in Kampala, Uganda
}

\author{
*Nakibuuka $\mathrm{VK}^{1}$, Okong $\mathrm{P}^{2}$, Waiswa $\mathrm{P}^{3}$, Byaruhanga $\mathrm{RN}^{1}$
}

1. Nsambya Hospital, Department of Paediatrics, Kampala, Uganda

2. Nsambya Hospital, Department of Obstetrics and Gyanecology, Kampala, Uganda

3. Makerere University, School of Public Health, Kampala, Uganda and Department of Global Health, IHCAR, Karolinska Institutet, Sweden

\begin{abstract}
Background: The perinatal mortality of 70 deaths per 1,000 total births in Uganda is unacceptably high. Perinatal death audits are important for improvement of perinatal care and reduction of perinatal morality. We integrated perinatal death audits in routine care, and describe its effect on perinatal mortality rate at Nsambya Hospital.

Methods: This was a retrospective descriptive study conducted from March - November 2008. An interdisciplinary hospital team conducted weekly perinatal death reviews. Each case was summarized and discussed, identifying gaps and cause of death. Local solutions were implemented according to the gaps identified from the audit process.

Results: Of the 350 perinatal deaths which occurred, 120 perinatal deaths were audited. $34.2 \%$ were macerated still births, $31.7 \%$ fresh still births and 34.2\% early neonatal deaths. Avoidable factors included: poor neonatal resuscitation skills, incorrect use of the partographs and delay in performing caesarean sections. Activities implemented included: three skills sessions of neonatal resuscitation, introduction of Continuous positive airway pressure (CPAP) for babies with respiratory distress, updates on use of partographs. Perinatal mortality rate was 47.9 deaths per 1000 total births in 2008 after introduction of the audits compared to 52.8 per 1,000 total births in 2007.

Conclusion: Conducting routine perinatal audits is feasible and contributes to reduction of facility perinatal mortality rate. Key words: perinatal, audits

African Health Sciences 2012; (4): 435 - 442 http://dx.doi.org/10.4314/ahs.v12i4.6
\end{abstract}

\section{Introduction}

It is estimated annually that there are 4 million neonatal deaths, approximately 3.3 million stillbirths and $99 \%$ of these deaths occur in low -income and middle income countries ${ }^{1}$.

In Uganda the perinatal mortality rate of 70 deaths per total 1000 births is unacceptably high. Although majority of the deliveries occur at home, substandard obstetric care in the different hospitals continues to be a cause of maternal and newborn morbidity and mortality ${ }^{2}$. Hospital-based studies in low income countries have shown that up to 3 out of 4 perinatal deaths may be due to suboptimal care ch $^{3,4}$.

Clinical audit is an important strategy in reducing health facility deaths. It is defined as a systematic critical analysis of the quality of medical care in order to identify mistakes, improve care including the procedures used for diagnosis, treatment, the use

\author{
*Corresponding author: \\ Victoria K Nakibuuka \\ Department of Paediatrics \\ Nsambya Hospital \\ P.o Box 7146 \\ Kampala, Uganda \\ Telephone: 0772369325 \\ E mailnnakibuukarv@yahoo.com
}

of resources and the resulting outcome and quality of life ${ }^{5}$. Several studies have demonstrated the contribution of perinatal death audits in improving quality of health care in resource limited settings ${ }^{6-8}$. Perinatal death audits reduce perinatal deaths in health facilities by $30 \%$. However, perinatal death audits have not yet been scaled up in Sub- Saharan Africa?. In Uganda, perinatal death audits are performed in only a handful of health facilities. The aim of this study was to integrate audit as routine instrument for quality improvement in medical care and to describe its effect on the perinatal mortality rate.

\section{Study site}

This was a retrospective descriptive study using perinatal death audit in San Raphael of St. Francis hospital Nsambya, a 361 bed private not for profit hospital in Kampala city, Uganda. The catchment area is Makindye West Health Sub-District with a population of 250,000 but the hospital also receives referrals from other facilities in and around Kampala district. The number of annual deliveries is 7200 and a maternal mortality ratio of 350 per 100,000 live births. The perinatal mortality rate in 2000 was 68.3 per 1000 total births ${ }^{10}$. The department of Obstetrics 
and Gyneacology has four consultants, three medical officers, four intern doctors and 30 midwives. The unit offers routine obstetric and gynaecological outpatient and inpatient care. Emergency obstetric care is available 24 hours, seven days a week all year round. Basic tools available include: blood pressure machines, feto-stethoscopes, partographs, delivery sets, vacuum extractors, caesarean section sets and newborn resuscitation equipment. In addition, there is a functional theatre with anesthetists available throughout the week.

There are three paediatricians, four intern doctors and seven nurses taking care of sick neonates admitted in neonatal special care unit. An average of 2000 babies is admitted annually. The unit has the following basic equipment: Incubators, phototherapy, basic resuscitation equipment and Continuous positive airway pressure equipment (CPAP) for babies with respiratory distress. Ventilators and monitors and intensive care unit capacity are not available.

\section{Methods \\ Data collection}

Perinatal death audits using standardized tools and procedures were reintroduced in

January/February 2008 by the departments of Pediatrics and Obstetrics and Gynecology. This was motivated by the high burden of perinatal deaths in the Hospital. An interdisciplinary audit team comprising of four obstetricians, two pediatricians, five medical officers, Twenty midwives ,two administrators that would meet on a weekly basis was formed. The audit team was lead by senior obstetrician or pediatrician. A core team consisting of obstetricians and pediatricians reviewed ministry of health perinatal death audit guidelines and tools, adopted them and trained medical officers, nurses and midwives on perinatal death audits. A perinatal death was as defined according to the International Classification of Disease 10 as death of a fetus greater than a birth weight of $1000 \mathrm{~g}$ if measured, or a gestation age of at least 28 completed weeks or a body length(crown/heel) of at least $25 \mathrm{~cm}$ or more which included all macerated, fresh still births and neonatal deaths within one week after delivery ${ }^{11}$.

The number and location of perinatal deaths was compiled on a daily basis from daily clinical handover reports and from registers in the labor wards and neonatal special care unit.

\section{The audit feedback process}

The clinical records of women who lost their babies and of babies admitted in neonatal special care unit were retrieved by the medical officers for the audit process which was performed weekly. The audit meeting was chaired and modulated by the most senior obstetrician or Pediatrician, information about clinical care was extracted from the clinical records by the medical officers or residents but health providers were not identified by name. An audit form for each case was completed by one of the medical officers. For purposes of the audit process, 240 cases were excluded because of lack of time or lack of clinical case notes. The clinical case notes were missing in the records department because they had not yet been forwarded due to administrative delays in the accounts department.

The cases were discussed in detail, identifying cause of death, care provided judged against existing standards/protocols. The quality of care offered to the mother/baby was agreed by consensus classified into four categories namely: 1.Optimum 2, Probably acceptable, 3. Probably suboptimal and 4. Suboptimal care. The Grading followed was similar to the grading of the Confidential Enquiries into stillbirths and deaths in infancy (12). No clinical autopsy were performed because there are costly and this is further compounded by the absence of a full time - pathologist at the hospital.

A detailed operational definition of these categories is given below;

$1=$ Optimal care generally accepted practice and all standards of care followed.

$2=$ Suboptimal care but different management would have made no difference to the outcome (Probably acceptable)

$3=$ Suboptimal care where different management might have made a difference to the outcome - an avoidable factor of uncertain clarity or influence on the outcome (probably suboptimal)

$4=$ Different management would reasonably be expected to have made a difference to the Outcome .A clearly avoidable factor implying that the adverse outcome could have been Prevented.) (Suboptimal)

Management policies on maternal and newborn care were based on evidence based interventions and National Ministry of health guidelines on maternal and newborn care ${ }^{13,14}$.

The suboptimal factors, grading and suggestions for change were documented on the audit tool during the meeting by the pediatrician or obstetrician. 
Because of many perinatal deaths and limiting audit meetings to only one hour all perinatal deaths were not audited each week. But there was purposive selection of cases to be audited from each of the following categories: macerated still births, fresh still births and early neonatal deaths; one third of the total audited from each category.

A summary of each case included a resolution of action to be taken and this was recorded in the minutes of the meeting in the attendance register and a person appointed to lead implementation of the action. On a monthly basis, implementation of resolutions was reviewed by the core team.

\section{Data management}

The quantitative data from audit forms was entered with Epidata version 7 and analyzed using SPSS version 12. Standard descriptive analysis was done to compute proportions. Narrative description of resolutions and activities to be implemented were made from minutes of the audit meetings.

\section{Ethical approval}

Ethical approval was obtained from Nsambya Hospital Ethical committee

\section{Results}

The baseline characteristics of the respondents are shown on table 1.

In a period of nine (9)months, a total of 6,041 deliveries were registered and there were 350 perinatal deaths, giving a perinatal death prevalence of $5.8 \%$. Only $34 \%(n=120)$ of all perinatal deaths were audited, of which $41(34.2 \%)$ were macerated still births, $38(31.7 \%)$ fresh still births and 41 (34.2 $\%$.) Neonatal deaths.

The mean maternal and gestational age at booking was 27 years and 22 weeks respectively.

A total of $109(90.2 \%)$ booked for antenatal visit while only $11(9.8 \%)$ did not. A total $6(5 \%)$ were HIV positive; however a large proportion $72(60 \%)$ did not have the HIV test done. A total of 57 (47.5\%) mothers received IPT while 63 (52.5\%) did not.
Table 1: Characteristics of mothers who had perinatal deaths

\begin{tabular}{|c|c|c|}
\hline Characteristic & Number & $\%$ \\
\hline Age (Mean in years) & $27.4 \pm 5.0$ & \\
\hline$\leq 20$ & 10 & $8.3 \%$ \\
\hline 21 to 34 & 101 & $84.2 \%$ \\
\hline$\geq 35$ & 9 & $7.5 \%$ \\
\hline \multicolumn{3}{|l|}{ Parity } \\
\hline$<1$ & 45 & $37.5 \%$ \\
\hline 2 to 5 & 68 & $56.7 \%$ \\
\hline$>5$ & 7 & $5.8 \%$ \\
\hline \multicolumn{3}{|l|}{ Attendance in ANC } \\
\hline Booked & 109 & $90.8 \%$ \\
\hline Un-booked & 11 & $9.2 \%$ \\
\hline \multicolumn{3}{|c|}{ Average number of ANC visits 5} \\
\hline Gestational age $1^{\text {st }}$ visit & $22 \pm 7.8$ & \\
\hline \multicolumn{3}{|c|}{$\begin{array}{l}\text { Intermitent malaria prophylactic treatment } \\
\text { (IPT) }\end{array}$} \\
\hline Yes & 57 & $47.5 \%$ \\
\hline No & 63 & $52.5 \%$ \\
\hline \multicolumn{3}{|l|}{ IPT by dosage } \\
\hline $1^{\mathrm{ST}}$ dose & 31 & $25.8 \%$ \\
\hline $2^{\text {nd }}$ dose & 21 & $17.5 \%$ \\
\hline $3^{\text {rd }}$ dose & 5 & $4.2 \%$ \\
\hline \multicolumn{3}{|l|}{ HIV Status } \\
\hline Positive & 6 & $5.0 \%$ \\
\hline Negative & 22 & $18.3 \%$ \\
\hline Test not done & 72 & $60.0 \%$ \\
\hline Results not given & 20 & $16.7 \%$ \\
\hline \multicolumn{3}{|l|}{ Type of pregnancy } \\
\hline Single & 117 & $97.5 \%$ \\
\hline Multiple & 3 & $2.5 \%$ \\
\hline \multicolumn{3}{|c|}{ Fetal heart present at admission } \\
\hline Yes & 73 & $60.8 \%$ \\
\hline No & 47 & $39.1 \%$ \\
\hline
\end{tabular}

The complications identified during pregnancy included; Hypertensive disorders and malaria were more prevalent than other conditions (table 2).

In a half of mother baby pairs, the quality of care was judged to be optimum and in nearly one third it was judged to be acceptable. One in five was judged to be probably or sub-optimum (figure 1).

Approximately one third of the total audited, the cause was unknown, autopsy is not routinely performed. Asphyxia is the single most important cause of death reported for 35 cases $(29.4 \%)$ of the perinatal deaths (table 3). 
Table 2: Complications during pregnancy

\begin{tabular}{lcr}
\hline Complication & $\begin{array}{c}\text { Number of } \\
\text { patients }\end{array}$ & Percentage \\
\hline Hypertensive disorders & 12 & $10.0 \%$ \\
Malaria & 8 & $6.7 \%$ \\
Abruptio placenta & 6 & $5.0 \%$ \\
Urinary Tract Infections (UTIs) & 4 & $3.3 \%$ \\
Prolonged rupture of membranes & 2 & $1.7 \%$ \\
Diabetes & 2 & $1.7 \%$ \\
$*$ Others & 12 & $10.0 \%$ \\
\hline
\end{tabular}

*Others include; Candida infections, Respiratory tract infections

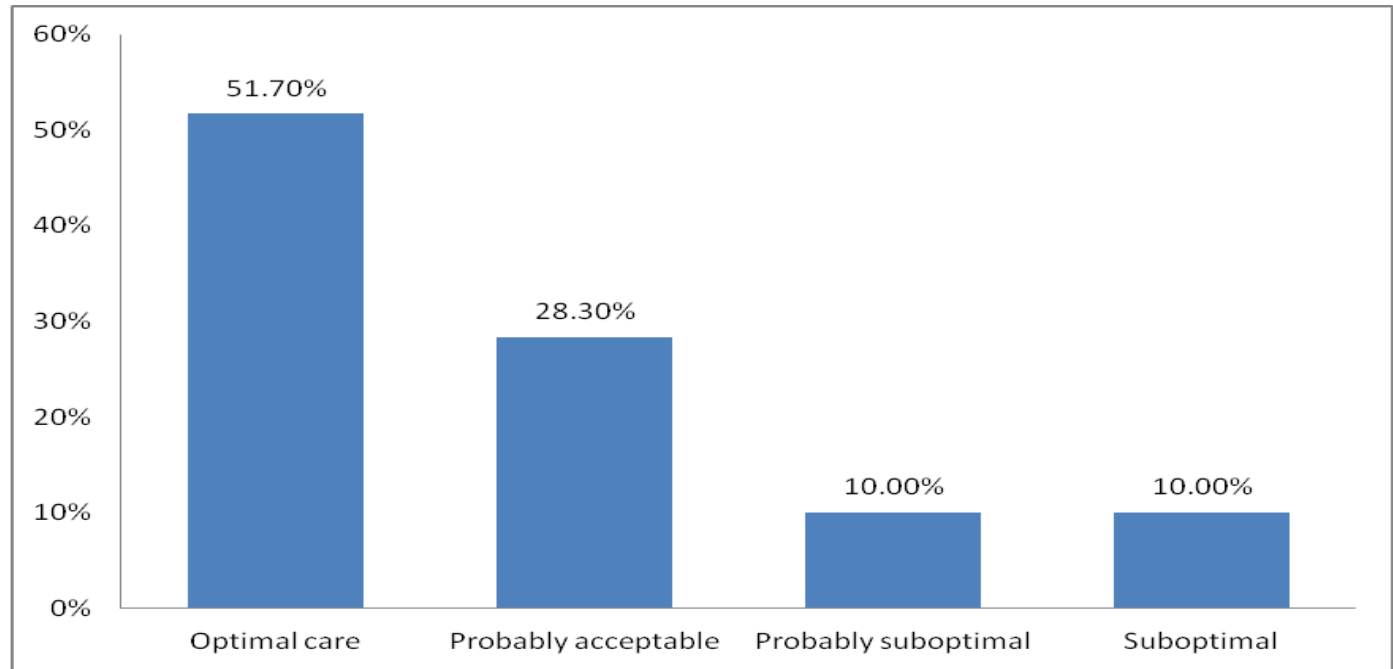

Figure 1: Quality of care offered to the mother and the baby

Table 3: Causes of perinatal deaths

\begin{tabular}{llr}
\hline Causes of death & Number & Percentage \\
\hline Unknown & 42 & $35.0 \%$ \\
Intrapartum Asphyxia & 35 & $29.2 \%$ \\
Others & 14 & $11.7 \%$ \\
Haemorrhagic disease of the new born & 13 & $10.8 \%$ \\
Respiratory distress Syndrome & 11 & $9.2 \%$ \\
Meconium aspiration Syndrome & 3 & $2.5 \%$ \\
Hypoxic Ishaemic encephalopathy & 2 & $1.7 \%$ \\
\hline
\end{tabular}

\section{Gaps in care that were identified}

Over the nine months periods, the following gaps were identified as major avoidable causes for suboptimal care (table 4).

\section{Table 4: Modifiable factors identified}

\begin{tabular}{lr}
\hline Modifiable factor & Number \\
\hline Surgery delayed for more than 30 minutes & 4 \\
Poor use of partograph & 3 \\
Poor resuscitation skills & 3 \\
Poor Management of diabetes in pregnancy & 1 \\
Poor management of PET & 1 \\
\hline
\end{tabular}


Three most important modifiable factors identified during the audit process were: delayed caesarean sections, poor use of Partograph and poor resuscitation skills.

\section{Surgery delay}

Four of the mothers that received suboptimal care had delayed surgery. It was found that after a decision was made to perform an emergency caesarean section the time interval between decisions to surgery was more than 30 minutes. This was attributable to a number of reasons namely: delay in pre-operative preparation in the ward, shortages of personnel in the labour wards or operating theatre, unavailability of operating space or sterile equipment.

\section{Partographs}

Incomplete or incorrect interpretation or lack of action. This accounted for three of the cases that received suboptimal care. Many midwives were vigilant in plotting their findings on the Partograph. However, careful review of the records at ward management level showed that this problem was linked to staff recently deployed in the labour wards (six months or less). There were also delays in interventions. The obstetric team was tasked to carry out update workshops for all midwives and doctors involved in labour management over a two months period.

\section{Substandard Resuscitation skills}

This accounted for three of the cases that received suboptimal care. The primary newborn care provider is a midwife. It was found that the majority of midwives and the doctors did not follow the protocol for newborn resuscitation. For example a baby with Apgar score 2 at 5 minutes it was commonly documented that only suction and dextrose $50 \%$ had been given during resuscitation. Ventilation with ambu-bag was not done routinely used even when a baby had no respiratory effort at birth. Sometimes the protocol had been adhered to but this was not documented.

\section{Inefficient management of diabetes mellitus in pregnancy}

Although only one of the cases that received suboptimal care had Diabetes mellitus. There was no clear protocol for identification of mothers at risk and management of mothers with Diabetes Mellitus in Pregnancy.

\section{Inefficient Management of eclampsia}

One of the mothers classified as having suboptimal care had Eclampsia. Although there is a protocol for preeclampsia management in the labour wards sometimes it is not followed adequately well.

\section{Addressing the quality of care gaps}

In the first nine months of implementing perinatal death audits many health system issues contributing to perinatal deaths were identified. However resolutions were made to implement the following activities to address problems identified during audits:

\section{Delays to implement surgical interventions: Caesarean sections}

Following the audit session the team agreed to reduce the time interval between decisions to Perform Caesarean section to actual surgery/delivery of the baby to 30 minutes. This resolution was made by the obstetric team comprising of nurses, midwives, medical officers and obstetricians. This new standard was disseminated to all the health workers involved. It was agreed that the doctors must be involved in pre-operative preparation of patients in the wards. Additionally more anaesthetists were recruited and it was suggested to the administration to build a maternity theatre.

\section{Incomplete or incorrectly interpreted partographs}

Updates on how to complete, interprete a partograph and actions were conducted for all midwives and doctors who had been deployed in the last six months.

\section{Poor neonatal resuscitation skills among} labour ward and neonatal special care unit staffs Three monthly training of Intern Doctors and midwives on neonatal resuscitation was done to address the problem of poor resuscitation skills. In addition there was provision of appropriate sizes of ambu-bags and masks in labour ward and newborn unit. Standard NRP neonatal resuscitation protocols were displayed in the labour wards and neonatal special care unit. Space for resuscitation consisting of a flat table, a firm baby mattress and heat source were set up in labour wards and special neonatal care unit. 
4. Management of respiratory distress syndrome using under bubble continuous positive

\section{Airway Pressure}

Starting in mid 2008, midwives in newborn unit were trained to recognize babies with respiratory distress and how to safely apply the nasal prongs and set up the local CPAP circuit. CPAP is a continuously applied distending pressure (CDP) used for maintenance of an increased trans-pulmonary pressure during expiratory phase of respiration, in a spontaneously breathing patient. This significantly reduces mortality in preterm babies with respiratory distress syndrome.

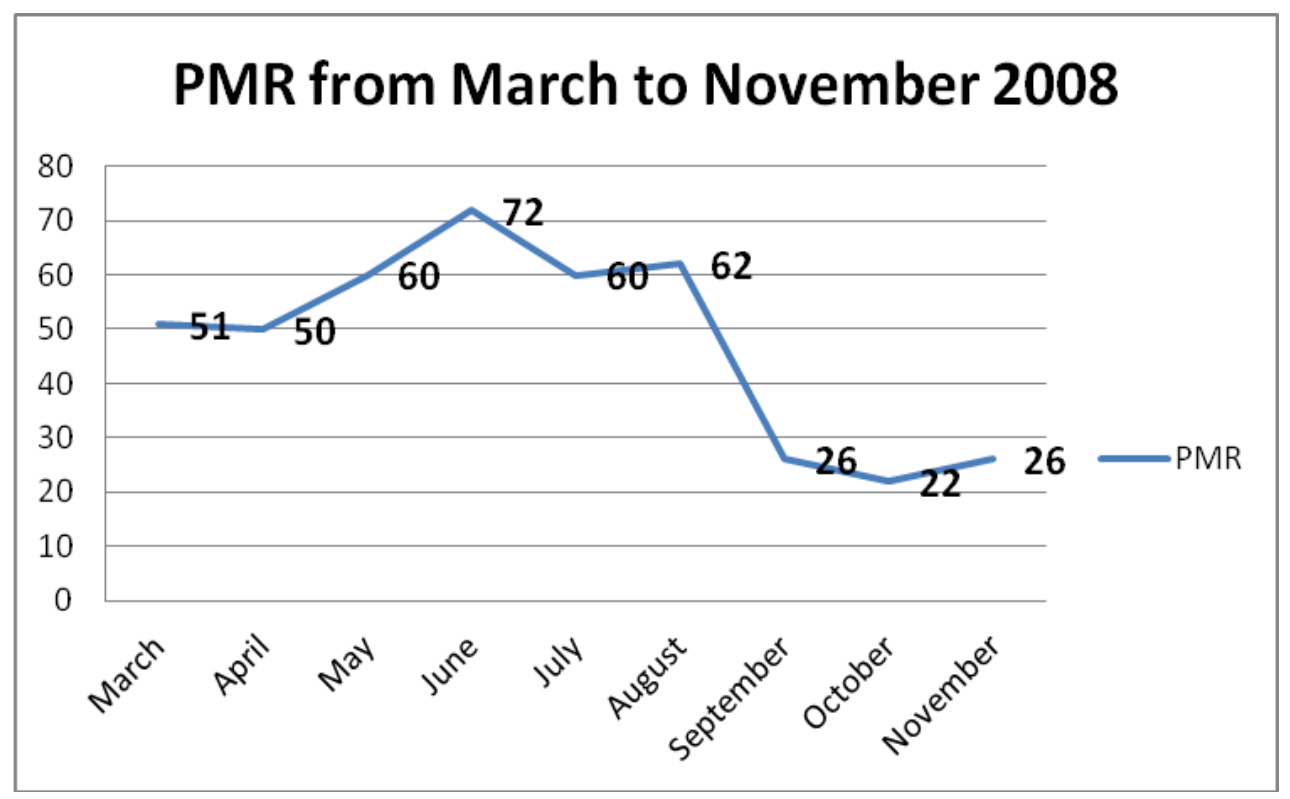

Figure 2: Monthly Perinatal mortality rate (9 months)

The figure above summarizes the perinatal mortality rate per month when audits were introduced in the hospital. The highest registered in June (72) and the lowest in October (22).

\section{Discussion}

This study demonstrates how to conduct perinatal death audits and implement actions to address systems failures to prevent perinatal deaths in a hospital setting. It also demonstrates the multidisciplinary team approach and no name and no blame audit process. This experience shows that perinatal audits can be introduced and become routine in low income settings such as in Uganda. In addition, the study shows that perinatal audits can contribute to health care quality improvement and reduce perinatal mortality.

In Uganda, like many other countries in Sub Saharan African countries, perinatal death audits are not yet routinely carried out in all health facilities for various reasons. This includes fear of health workers to be identified in person and blamed for the death, lack of participation of health facility managers and lack of implementation of actions to correct systems weaknesses. If no actions are implemented and 440 practice environment does not change, the health workers easily loose morale to carry out audits and might suffer burn out because of identifying the same issues and no action is taken. This study has been able to demonstrate that respecting to all these elements of perinatal death audits contributes to sustaining the practice.

In a period of nine months, weekly perinatal death audits were conducted. It was found that half the cases had optimum care and only $20 \%$ had suboptimal care. The findings for optimal care are consistent with the findings in Sudan $(43 \%)^{15}$. The findings of $20 \%$ had sub-optimal care Hlabisa is similar to findings from Health district in Kwazulu Natal (19\%), South Africa ${ }^{6}$. Using independent external auditors showed a higher proportion of cases were judged to have received sub-optimum care in Sudan (83\%), Tanzania (53\%) and U.K $(75 \%)^{6,15,16}$. This difference in proportions of cases judged to have received sub-optimum care by internal vs. external auditors might be that internal evaluators were less critical (protective) in their assessment. They might have been favorably lenient, given the fact that they were conversant with the local environment and the available limited resources. However the external 
auditors in other studies were from different clinical settings and background and this may have led to the different results of their assessment.

In this study the quality of care offered to the mother/baby was agreed to by consensus through open group discussions using standards/ protocols as a yard stick. It was not a decision of one individual and this differs from other studies (Tanzania and Sudan) in which decisions were taken by individuals ${ }^{15,16}$. This approach in this study was deemed important for its inclusiveness of all care team members and offers opportunity to learn. This further promoted ownership and sustainability of the audit process.

\section{Modifiable factors and Interventions}

Audit was used to identify some of the suboptimal factors and these included: poor resuscitation skills, substandard use of partograph and delayed surgery. These were the major factors associated with suboptimal care but perinatal death audits alone will not uncover all avoidable factors. It is necessary to implement other quality improvement strategies such as "near miss audits" and criteria based audits for specific pregnancy complications irrespective of outcomes. Household and community factors and these other quality interventions can help further reduce the number of perinatal deaths.

Over a period of nine months the audits covered only a sample of perinatal deaths. When there are many deaths, one must be realistic about the number that can be audited, and it is also known that if you audit many deaths some issues will be recurrent and you reach a point of saturation in trying to identify avoidable factors. In order to identify the causes of perinatal deaths it is necessary to carry out autopsies and to audit all deaths. This was not the objective of this nine months feasibility phase of audits. Also several health system weaknesses were identified but the team agreed to implement interventions to address only three of these. This was done for pragmatic purposes knowing what can actually be done rather than resolve to do many things and fail to implement them. The three interventions required organization of workshops to update knowledge and in other instances develop new skills. It is important to note that these were implemented, a rare accompaniment of an audit report.

A large number of our mothers (82.3\%) with macerated still births reported to hospital when the babies were already dead. The interventions chosen in the first nine months of audit did not address this group of perinatal deaths. Specific interventions for this group of perinatal deaths will be implemented in the future as the perinatal death audit process matures in the hospital. Also at a later stage or in the future, audits will try to identify household and community factors and propose some interventions.

\section{Effect on Perinatal mortality rate}

The overall Perinatal mortality rate (PMR) in 2008 was 47.9 compared to 52.8 per 1000 total births in 2007 and there was a subsequent reduction over the months the highest in June (72) and lowest (22) in October ( figure 2). The number and types of health workers did not change in the nine months period in these wards, neither were the work schedules altered. We attribute the reductions of perinatal death mortality rate to training and retraining of staff in the three identified interventions and procurement of equipment. This reduction in PMR is similar to the reports from Mozambique, Tanzania and South Africa; $40 \%, 32 \%$ and $50 \%$ respectively ${ }^{6,8,16}$. Even greater reductions in perinatal mortality rate was reported in Western Tanzania, from 71 to 39/1000 following introduction of perinatal death audits ${ }^{17}$. Similar effects were reported in Norway and Netherlands ${ }^{18,19}$.

\section{Limitations of the study}

Autopsies were not performed for all cases of perinatal deaths and all perinatal deaths were not audited due to lack of time and unavailability of records. Therefore it was not possible to describe all the causes of deaths accurately. The causes of perinatal deaths and even avoidable factors therefore cannot be generalized to perinatal deaths in other hospitals in Kampala or to the population.

Recommendations

1. Endeavour to recommend autopsies to parents who have lost a baby to improve on the epidemiology and causes of perinatal deaths.

2. Endeavour to audit more perinatal deaths to improve on the data base of the causes of perinatal deaths

3. Introduce criteria based audits for specific disease conditions even for women and babies who do not die, to compliment perinatal death audits for improvement of the quality of care. 


\section{Conclusion}

Health facility perinatal death audits can be successfully implemented as a routine activity and sustained by setting a specific day known to everyone, respecting time and avoiding blaming particular health workers. A core team is necessary to provide leadership and guidance for audit process and to ensure implementation of activities. It is necessary to start with a few activities to address health system failures and implement them to complete the audit cycle.

\section{Acknowledgements}

The health workers in the Obstetrics and Paediatric departments of Nsambya Hospital who work tirelessly to improve quality of maternal and newborn care.

\section{References}

1. Lawn JE, Cousen S, Zupan J. Neonatal Survival Steering team, 4 Million Neonatal Deaths, When , Where, why, . Lancet 2005;365:891-900.

2. Mbonye AK, Mutabazi MG, Asimwe JB, Sentumbwe O, Kabarangira J, Nanda G, et al. Declining maternal mortality ratio in Uganda :Priority interventions to achieve the Millennium Development Goal. International Journal of Gynaecology and Obstetrics 2007 .96:285-290.

3. Hinderaker SG, Olsen BE, Bergsjo PB, Gasheka P, Lie RT, Havnen J, et al. Avoidable stillbirths and neonatal deaths in rural Tanzania. BrJ Obstet Gynaeco 2003; 110:616-623.

4. Kidanto HL, Massawe SN, Nystrom L, Lindmark G. Analysis of perinatal mortality at a teaching hospital in Dar es Salaam, Tanzania, 1999-2003. Afr J Repr Health 2006; 10:72 -80.

5. Johnson G, Crombie IK, Davies HTO, Ailder EM, Millard A. Reviewing audit: barriers and facilitating factors for effective clinical audit. Quality in Health Care 2000; 9:23-26.

6. Wilkinson D. Reducing perinatal mortality in developing countries. Health Policy and Planning 1997;12:161-165.

7. Ward HRG, Howarth GR, Jenning OJN, Pattinson RC Audit incorporating avoidability and appropriate interventions can significantly decrease perinatal mortality. South African Medical Journal. 1995;85:147-50.

8. Bugalho A, Bergstrom S, The value of perinatal audit in Obstetrics Care in the developing world: A ten year experience of the Maputo Model. Gynaecological Obstetric investigation 1993;36:239-43.
9. Robert Pattison, Kate Keber, Peter Waiswa, Louise T.Day, Fecility Musell, Asiruddin Sk, et al. Perinatal Mortality Audit: Counting, accountability, and over coming challenges in scaling up in low-and middle income countries. International Journal of Gynaecology and Obstetrics 2009;109:113-122.

10. Byaruhanga RN. Improving healthcare by perinatal mortality audit and feedback. Trop Doct 2000;30(2):94-7.

11. World Health Organization. International Classification of Disease: World Health Organization; 2006.

12. Young P, Hamiliton R, Hodgett S, Moss M, Jones P, Johanson R. Reducing risk by improving standards of intra-partum fetal care. Journal of the Royal Society of medicine 2001;94:226-231.

13. Darmstadt G.L, Bhutta Z.A, Cousen S, Adam T, Bernis L. De, Walker N. Evidence - Based cost effective Interventions that matter, How many new borns can we save at what cost Lancet 2005;94631:977- 88.

14. World Health Organization. The WHO Reproductive Health Library. Geneva; 2007.

15. Seif El Al JL-R, Birght Bodker, Ali-AbuBakr, Abdu Latif, Ashmeig Sala A Ibrahim, Guinilla Lind Mark,. Introducing Qualitantive audit in a tertiary Hospital in Sudan. Health Policy and Planning 2002;17:296-303.

16. Kidanto H, Mogren I, Van Roosmalen J, Thomas NA, Massawe SN, Nystrom L, et al. Introduction of a qualitative perinatal audit at Muhimbili National Hospital, Dar-es- salaam, Tanzania. BMC, Pregnancy and Child Birth 2009;9:45-

17. Van Roosmalen J. Perinatal Mortality in Rural Tanzania. British Journal of obstetrics and Gynaecology 1989;96:827-834.

18. Babill Stray - Pedersen. Perinatal mortality in Norway: Experiences with audit. European Journal of obstretics and gynaecology and Reproductive Biology 1991; 41: 20-22.

19. Van Dillem, Marikie Hermus, Jacqueline of Hilligersberg, Peter Nikkels, Herman oosterbaan, et al. Netherlands Foundation for Perinatal Audit :2011 